\title{
Psychostimulants Differentially Regulate Serotonin Transporter Expression in Thalamocortical Neurons
}

\author{
Terri L. Whitworth, Laura C. Herndon, and Michael W. Quick \\ Department of Neurobiology, University of Alabama at Birmingham, Birmingham, Alabama 35294
}

5-HT transporters (SERTs) are transiently expressed in thalamocortical neurons during development, permitting these glutamatergic neurons to co-release 5-HT as a "borrowed" transmitter. The high level of SERT expression in these neurons is likely important in the serotonergic modulation of neocortical circuits and provides a system for examining endogenous SERT regulation. We tested the hypothesis that developmental expression of SERT in thalamocortical neurons is regulated by psychostimulants that are agonists and antagonists of SERT. Cultured thalamocortical neurons from embryonic day 18 rats were examined for SERT expression until P15. In untreated cultures, SERT protein levels peaked at postnatal day 3 (P3) and were absent by P10. Chronic treatment with SERT substrates (5-HT, 3,4-methylenedioxymethamphetamine) increased both peak SERT protein levels (fourfold) and the time course of SERT expression. SERT substrates also shifted the relative functional expression of SERT by redistributing intracellular SERT protein to the plasma membrane. The subcellular redistribution was prevented by PKC activators. SERT antagonists (e.g., fluoxetine, cocaine) reduced total SERT expression levels and the time course of SERT expression. These data (1) show that endogenous SERT is differentially regulated by $5-\mathrm{HT}$ and psychostimulants, (2) indicate that SERT modulation occurs via changes in both total SERT protein levels and subcellular redistribution of the transporter, and (3) suggest that some of the actions of drugs of abuse in neocortical development may be attributable to alterations in SERT expression and concomitant changes in 5-HT signaling.

Key words: cortex; development; drug abuse; monoamine; regulation; uptake
The cerebral cortex is innervated by serotonergic afferents during early stages of development, suggesting that this modulatory system plays an important role in the formation of the intricate neural circuits that comprise the mammalian neocortex (Buznikov and Shmukler, 1981; Lauder, 1993). In rodents, 5-HT immunocytochemistry reveals dense cortical 5-HT staining that disappears within the first several postnatal weeks (D'Amato et al., 1987). Some of this innervation comes from 5-HT neurons originating in the raphe (Bennett-Clarke et al., 1993). However, the spatial and temporal pattern of 5-HT immunoreactivity correlates directly with the expression of 5-HT transporters found on synaptic vesicles (Lebrand et al., 1998) and neuronal plasma membranes (Hansson et al., 1998; Lebrand et al., 1998) of nonserotonergic neurons. These transporters have been localized to glutamatergic thalamocortical afferents (Lebrand et al., 1996; Bruning and Liangos, 1997). Because the machinery to synthesize 5-HT is not present in these glutamate-containing neurons, it is suggested that thalamocortical afferents "borrow" 5-HT via transporter uptake and release it during the several postnatal weeks in which SERT is expressed (Lebrand et al., 1996).

Thus, factors that regulate SERT expression on these thalamocortical afferents would play a significant role in cortical development. SERT expression can be regulated by several different triggers, including receptor stimulation (Launay et al., 1994; Miller and Hoffman, 1994) and kinase activity (Jayanthi et al.,

Received July 9, 2001; revised Sept. 25, 2001; accepted Oct. 3, 2001.

This work was supported by National Institutes of Health Grants HD38760 and DA10509 and by a National Alliance for Research on Schizophrenia and Depression Young Investigator Award to M.W.Q.

Correspondence should be addressed to Michael W. Quick, Department of Neurobiology, CIRC 446, University of Alabama at Birmingham, 1719 Sixth Avenue South, Birmingham, AL 35294-0021. E-mail: quick@nrc.uab.edu.

Copyright (C) 2001 Society for Neuroscience $0270-6474 / 01 / 210001-05 \$ 15.00 / 0$
1994; Yura et al., 1996). The activation of protein kinase C directly phosphorylates SERT (Ramamoorthy et al., 1998), and this activation correlates with an internalization of the transporter (Qian et al., 1997). SERTs are the site of action of a number of psychostimulants that are transporter substrates (amphetamine) or antagonists (cocaine, fluoxetine). In heterologous expression systems, these drugs regulate surface SERT expression (Ramamoorthy and Blakely, 1999). In the present experiments, we test the hypothesis that SERT agonists and antagonists differentially regulate the expression of endogenous SERT on glutamatergic, thalamocortical neurons during development.

\section{MATERIALS AND METHODS}

Chemicals and reagents. All general cell culture reagents were obtained from Life Technologies (Gaithersburg, MD). Papain was obtained from Worthington (Freehold, NJ). Radiolabeled compounds and immunoblot detection reagents were obtained from Amersham Pharmacia Biotech (Arlington Heights, IL). All antibodies were obtained from Chemicon (Temecula, CA). Biotinylation reagents were obtained from Pierce (Rockford, IL). Vectashield was obtained from Vector Laboratories

This article is published in The Journal of Neuroscience, Rapid Communications Section, which publishes brief, peerreviewed papers online, not in print. Rapid Communications are posted online approximately one month earlier than they would appear if printed. They are listed in the Table of Contents of the next open issue of JNeurosci. Cite this article as: JNeurosci, 2001, 21:RC192 (1-6). The publication date is the date of posting online at www.jneurosci.org.

http://www.jneurosci.org/cgi/content/full/5931 
(Burlingame, CA). All other drugs and reagents were obtained from Sigma.

Cell culture and uptake assays. Primary cultures of thalamocortical neurons were prepared from Sprague Dawley rat embryos at age embryonic day 18 (E18). The thalamus was dissected and then minced in HEPES-buffered HBSS without calcium and magnesium. Tissue was digested in papain solution (Earle's balanced salt solution, $\mathrm{pH} 7.3,20$ $\mathrm{U} / \mathrm{ml}$ papain, $1 \mathrm{~mm}$ L-cysteine, $0.5 \mathrm{~mm}$ EDTA, and $0.1 \mathrm{U} / \mathrm{ml}$ DNase I). Tissue was incubated for $20 \mathrm{~min}$ at $37^{\circ} \mathrm{C}$ followed by gentle trituration, dilution, and plating. To obtain pure neuronal cultures, mixed cultures were treated for $48 \mathrm{hr}$ with $10 \mu \mathrm{M}$ cytosine arabinoside; treatment was initiated $24 \mathrm{hr}$ after plating. Cells were maintained in Earle's MEM supplemented with $10 \%$ FBS. Drugs and medium were added from the time of plating and replaced every $6 \mathrm{hr}$ for the duration of the experiment; in experiments not requiring drug application, medium was changed every $24 \mathrm{hr}$. Uptake assays were performed as described previously (Bernstein and Quick, 1999). The final $\left[{ }^{3} \mathrm{H}\right] 5-\mathrm{HT}$ concentration of the assay solution was $40 \mathrm{nM}$; the total 5 -HT concentration of the assay solution was $30 \mu \mathrm{M}$. To minimize changes in transporter expression during the assay, assay times were $10 \mathrm{~min}$. Before assay, cells were washed five times with $2 \mathrm{ml}$ of HBSS to minimize residual effects of chronic drug treatments on 5-HT uptake. This protocol was chosen because it yielded statistically equivalent uptake results in untreated cultures and cultures treated for $24 \mathrm{hr}$ with $300 \mu \mathrm{M}$ cocaine (data not shown). Statistical analyses of the uptake data were performed using SPSS (Chicago, IL).

Immunocytochemistry and confocal microscopy. For immunostaining experiments, neurons were plated on glass coverslips pretreated with poly-L-lysine $(10 \mu \mathrm{g} / \mathrm{ml})$ and fixed with $4 \%$ paraformaldehyde. After three washes with PBS, cells were permeabilized for $10 \mathrm{~min}$ in PBS containing $0.1 \%$ Triton X-100 incubated for $1 \mathrm{hr}$ in blocking solution (PBS, $1 \%$ bovine serum albumin, and $5 \%$ goat serum). Neurons were incubated with primary antibodies (anti-SERT, 1:2000; anti-glutamate, 1:1000) for $1 \mathrm{hr}$ at room temperature followed by $30 \mathrm{~min}$ incubation with secondary antibodies (FITC-conjugated IgG; Texas Red-conjugated IgG). Cells were then washed five times in blocking solution, and the coverslips were mounted on glass slides using Vectashield. Fluorescent images were generated using a Zeiss confocal microscope at $498 \mathrm{~nm}$ for FITC and $585 \mathrm{~nm}$ for Texas Red.

Biotinylation and immunoblotting. Biotinylation experiments were performed as described previously (Qian et al., 1997; Davis et al., 1998). The supernatant fractions $(300 \mu \mathrm{l})$ were incubated with an equal volume of Immunopure Immobilized Monomeric Avidin beads at room temperature for $60 \mathrm{~min}$. The beads were washed three times with RIPA buffer, and adsorbed proteins were eluted with SDS sample buffer $(62.5 \mathrm{~mm}$ Tris-Cl, pH 6.8, 2\% SDS, $100 \mathrm{~mm} \beta$-mercaptoethanol) at room temperature for $30 \mathrm{~min}$. Samples were run on a $6 \%$ acrylamide gel and blotted onto nitrocellulose membrane. For biotinylation experiments, analysis was performed on aliquots (1) taken before incubation with beads (total cell lysate), (2) of the supernatant fraction after adsorption and centrifugation (intracellular fraction), and (3) of the bead eluate (biotinylated fraction). Western blotting was performed as described previously (Corey et al., 1994) using anti-SERT antibodies (1:500); secondary antibodies were conjugated with HRP, and immunoreactive bands were detected with enhanced chemiluminescence. To ensure equal amounts of protein were being assayed in each condition, SERT levels were quantified relative to actin levels in the same experiment. Immunoreactive bands were scanned and quantified with ImageQuant (Molecular Dynamics, Sunnyvale, CA).

\section{RESULTS}

To verify that SERT was expressed in developing thalamocortical neurons in culture, dissociated thalamic neurons from E18 rats were plated and examined at postnatal day $3(\mathrm{P} 3)$ using confocal immunofluorescence microscopy (Fig. $1 A$ ). At $\mathrm{P} 3,>90 \%$ of the neurons that were immunoreactive for glutamate were also immunoreactive for SERT. SERT antibody preabsorbed with a C-terminal SERT peptide blocked all SERT immunoreactivity in these cells (data not shown). To examine the time course of SERT expression during early postnatal development in these neurons, we performed immunoblot experiments on thalamic neurons cultured at E18 and compared the amount of SERT immunoreactivity at six different early postnatal ages (Fig. 1B).

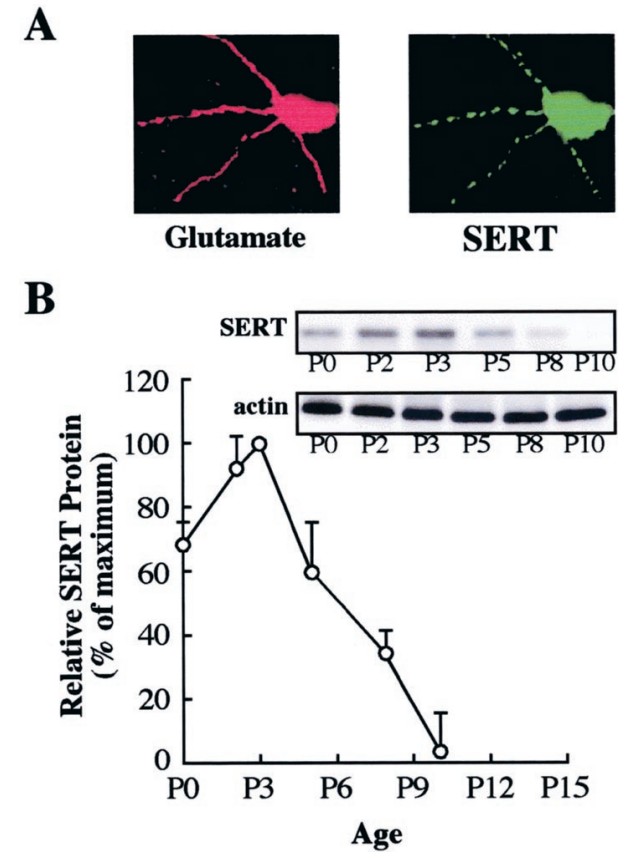

Figure 1. SERT is expressed in thalamocortical neurons during development. $A$, SERT colocalizes with glutamate. P3 neurons were stained with an anti-glutamate antibody conjugated to Texas Red and a C-terminal anti-SERT antibody conjugated to FITC. B, SERT expression is transient. Total cellular SERT protein levels were quantified with respect to maximal SERT expression (P3). Data are from five separate experiments. The inset shows a representative immunoblot from one experiment. Densitometry measurements of SERT expression (top blot) were adjusted relative to actin immunoreactivity (bottom blot) measured in the same experiment.

SERT protein levels increased to a peak at P3 and declined thereafter; SERT immunoreactivity was measurable at P8 but absent by P10. These results differed markedly from measurements of actin immunoreactivity, which remained relatively constant over this same time period. Single experiments conducted on neurons cultured until P21, P28, or P33 failed to show any SERT immunoreactivity (data not shown). These data in culture are very similar to previous results performed in brain using radiolabeled citalopram binding, 5-HT immunocytochemistry, and mRNA quantification (D'Amato et al., 1987; Dori et al., 1996; Lebrand et al., 1998).

Because SERT expression is one component necessary for thalamocortical neurons to release 5-HT (Lebrand et al., 1996), regulation of SERT expression should have a significant effect on 5-HT regulation of cortical development. The high expression of SERT in thalamocortical cells in culture allowed us to examine endogenous SERT regulation in neurons. Because SERT can be regulated in expression systems by agonists and antagonists (Ramamoorthy and Blakely, 1999), we examined the role of such molecules on SERT expression in thalamocortical neurons. E18plated cultures were chronically treated with $10 \mu \mathrm{M} 5-\mathrm{HT}$, and protein levels were assessed at subsequent time points by total cell SERT immunoreactivity (Fig. 2A). Compared with untreated control cultures, 5-HT-treated cultures showed two differences: (1) total SERT protein levels were approximately fourfold higher, and (2) both the peak of SERT expression and the loss of SERT immunoreactivity were shifted to older ages by several days. The increase in total SERT protein levels was 5-HT concentrationdependent (Fig. 2B), with half-maximal increases in total SERT 
A
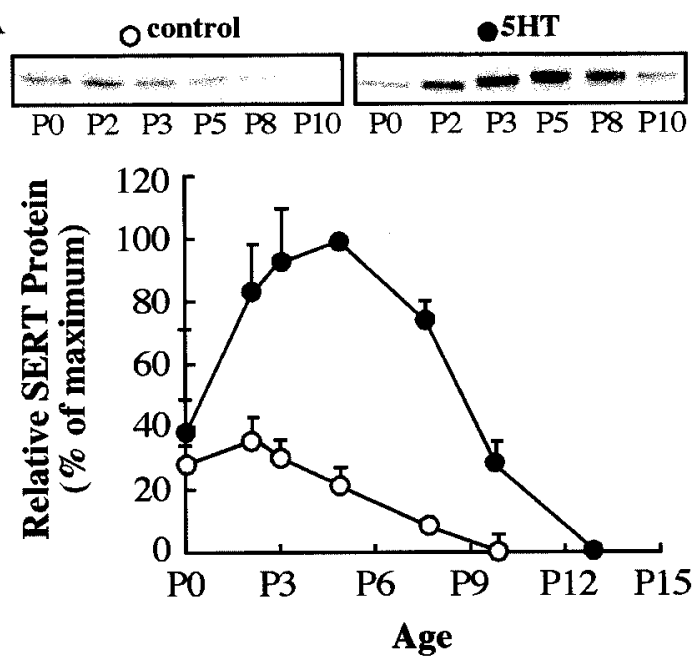

C

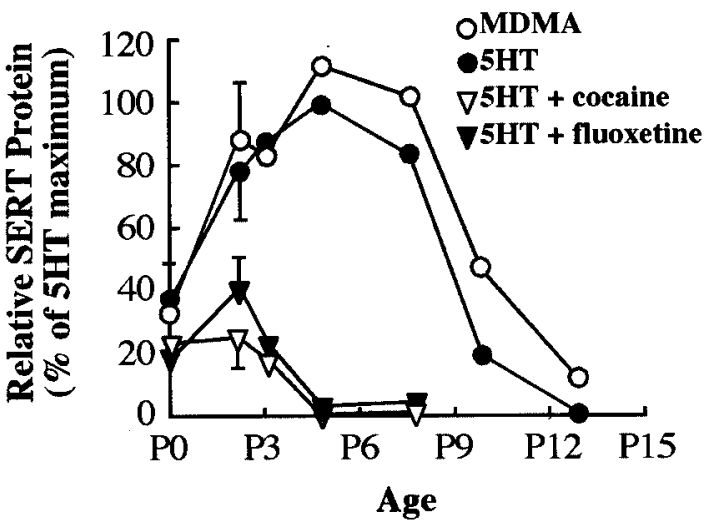

B

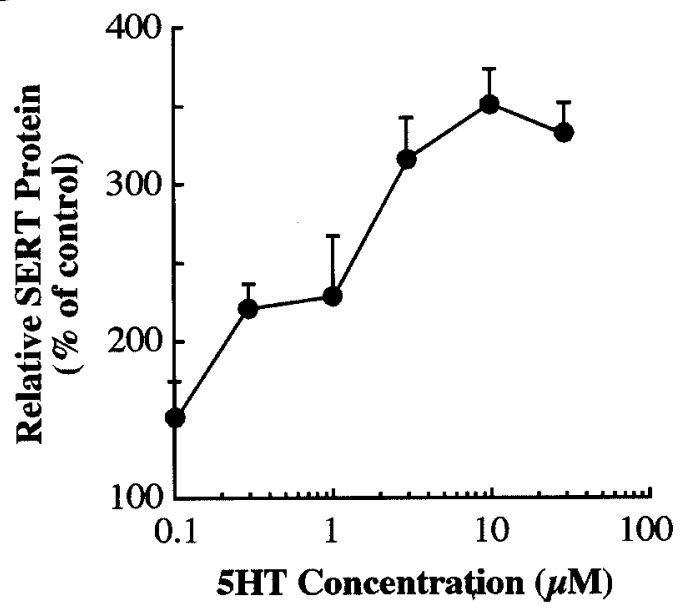

D

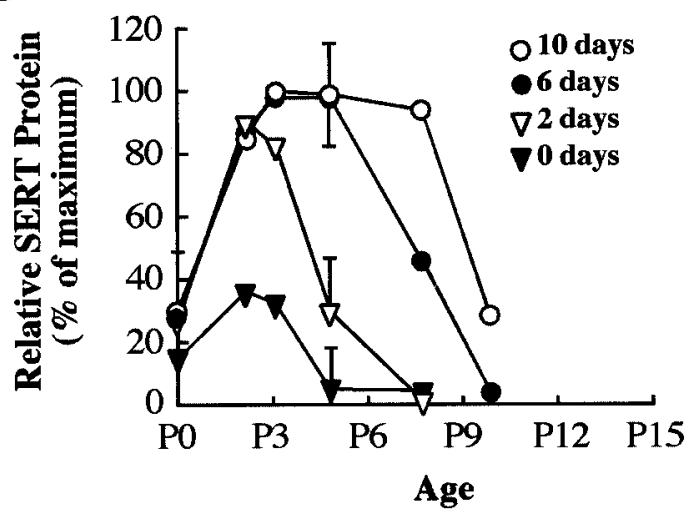

Figure 2. SERT agonists and antagonists differentially regulate the time course of SERT expression. A, 5-HT regulates total SERT proteins levels. Cultures were treated from time of plating with $10 \mu \mathrm{M}$ 5-HT. Total cellular SERT protein levels for untreated and 5-HT-treated cultures were quantified relative to maximal SERT expression (5-HT-treated neurons at P5). Data are from four separate experiments. The inset shows representative SERT immunoreactivity from one experiment. $B, 5$-HT regulation of total SERT protein levels is concentration-dependent. Cultures were treated with different concentrations of 5-HT and assayed at P3. Data are from three separate experiments and plotted relative to untreated cultures. $C$, SERT agonists and antagonists have differential effects on total SERT protein levels. Cultures were treated with the following drug concentrations (in $\mu \mathrm{M}$ ): 10 -HT, 10 MDMA, 300 cocaine, and 300 fluoxetine. Total cellular SERT protein levels quantified relative to SERT expression of 5-HT-treated cultures at P5. Data are from four separate experiments. Average SEs across all ages for each treatment group are summarized by the error bars plotted at P3. D, Time course of 5-HT effects on total SERT protein levels. Cultures were treated with $10 \mu \mathrm{M} 5$-HT for four different lengths of time from the time of plating. Data are from three separate experiments. Average SEs across all ages for each treatment group are summarized by the error bars plotted at P5.

protein levels assessed at P3 occurring at $\sim 1 \mu \mathrm{M}$. This is near the apparent affinity of SERT for 5-HT transport (Blakely et al., 1991; Hoffman et al., 1991).

SERT is the site of action for drugs of abuse such as 3,4methylenedioxymethamphetamine (MDMA) and cocaine, and for therapeutic drugs such as fluoxetine. E18-plated cultures were chronically treated with these compounds, and protein levels were assessed at subsequent time points by examining total cell SERT immunoreactivity (Fig. 2C). MDMA, a SERT substrate (Rudnick and Wall, 1992), regulated SERT expression similarly to 5-HT. Cotreatment of cultures with 5-HT and either of the SERT antagonists cocaine or fluoxetine eliminated both the fourfold increase in peak SERT expression and the increase in expression time course mediated by 5-HT. The time course of SERT immunoreactivity in the presence of cocaine and fluoxetine was decreased compared with untreated controls (compare with Fig. $2 A$ ). This could be caused by specific action of these compounds on SERT expression; however, it may also indicate that in untreated cultures there is some uptake of 5-HT from nominally
5-HT-free culture media. The continued presence of 5-HT was required to maintain the increased time course of SERT expression (Fig. 2D): removal of substrate at a given age resulted in a noticeable decline in SERT expression soon thereafter. In summary, these data suggest that the effect of 5-HT on SERT protein expression is mediated through actions on SERT, that SERT agonists increase and SERT antagonists decrease the magnitude and time course of SERT protein expression respectively, and that the continued presence of substrate is necessary for maintaining the increase in SERT expression.

The ability of thalamocortical neurons to use 5-HT as a "borrowed" transmitter requires the expression of functional SERTs. To examine functional SERT expression, we performed $\left[{ }^{3} \mathrm{H}\right] 5$ HT uptake assays on cultures chronically treated with MDMA alone or MDMA in the presence of cocaine (Fig. $3 A$ ). Assays were performed in the presence of 5-HT alone. Qualitatively, 5-HT uptake at different ages resembled that for total SERT protein levels. That is, MDMA-treated cultures showed 5-HT uptake that was increased in both magnitude and time course 
Figure 3. MDMA regulates the subcellular distribution of SERT. $A$, MDMA upregulates SERT function. Cultures were treated from time of plating with $10 \mu \mathrm{M}$ MDMA or $10 \mu \mathrm{M}$ MDMA plus $300 \mu \mathrm{M}$ cocaine. Uptake data are from three separate experiments, three wells per data point per experiment. $B, 5$-HT uptake is upregulated by MDMA to a greater extent than total protein levels. At each age, uptake and total cellular protein levels were determined from the same cultures and plotted as the ratio of values obtained for MDMA-treated versus MDMA plus cocaine-treated cultures. Data are from two separate experiments. $C$, MDMA causes a subcellular redistribution of SERT protein. Surface biotinylation experiments were performed on cultures from two different ages (P0, P5) treated from time of plating with $10 \mu \mathrm{M}$ MDMA $(M)$ or $10 \mu \mathrm{M}$ MDMA plus $300 \mu \mathrm{M}$ cocaine $(M+$ $C)$. Representative immunoblots examining SERT and $\mathrm{Na} / \mathrm{K}$ ATPase immunoreactivity are shown. $D$, Quantification of SERT expression from experiments as performed in $C$. The amount of biotinylated (surface) and nonbiotinylated (intracellular) SERT is plotted as percent of total SERT. Data are from three separate experiments. Dashed lines indicate the average amount of surface SERT in the presence of MDMA and in the presence of MDMA plus cocaine. Asterisks indicate significant differences between treatment groups $(p<0.05)$. $E$, Time course of agonist-induced subcellular redistribution of SERT. Untreated cultures at P3 were acutely treated with $10 \mu \mathrm{M}$ MDMA or $10 \mu \mathrm{M}$ MDMA plus $300 \mu \mathrm{M}$ cocaine for the time indicated on the abscissa; cultures were then subjected to surface biotinylation. Data are from two separate experiments. Asterisks indicate significant differences between treatment groups at particular time points $(p<0.05) . F$, The subcellular redistribution of SERT by MDMA is prevented by pretreatment with PKC activators. Cultures were treated from time of plating with 10 $\mu \mathrm{M}$ MDMA in the presence of $1 \mu \mathrm{M}$ PMA or $4 \alpha \mathrm{PDD}$ and assayed by biotinylation at P0. Data are from three separate experiments. Asterisk indicates significant differences between treatment groups $(p<0.05)$.
$\mathbf{A}$

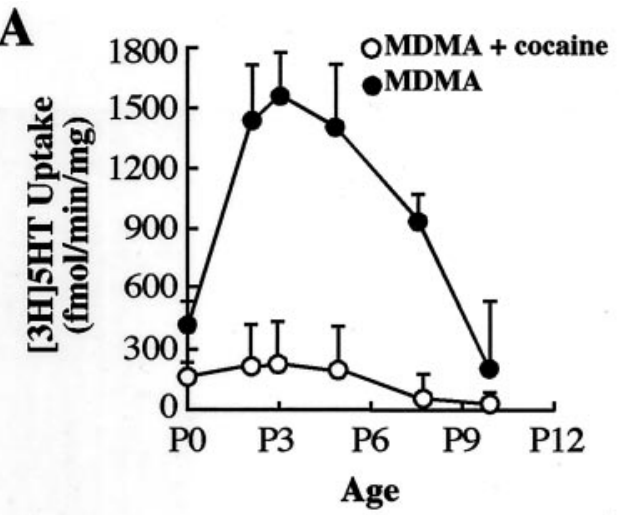

C

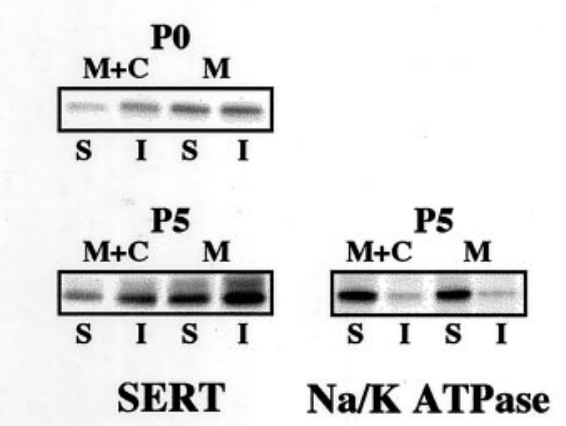

D
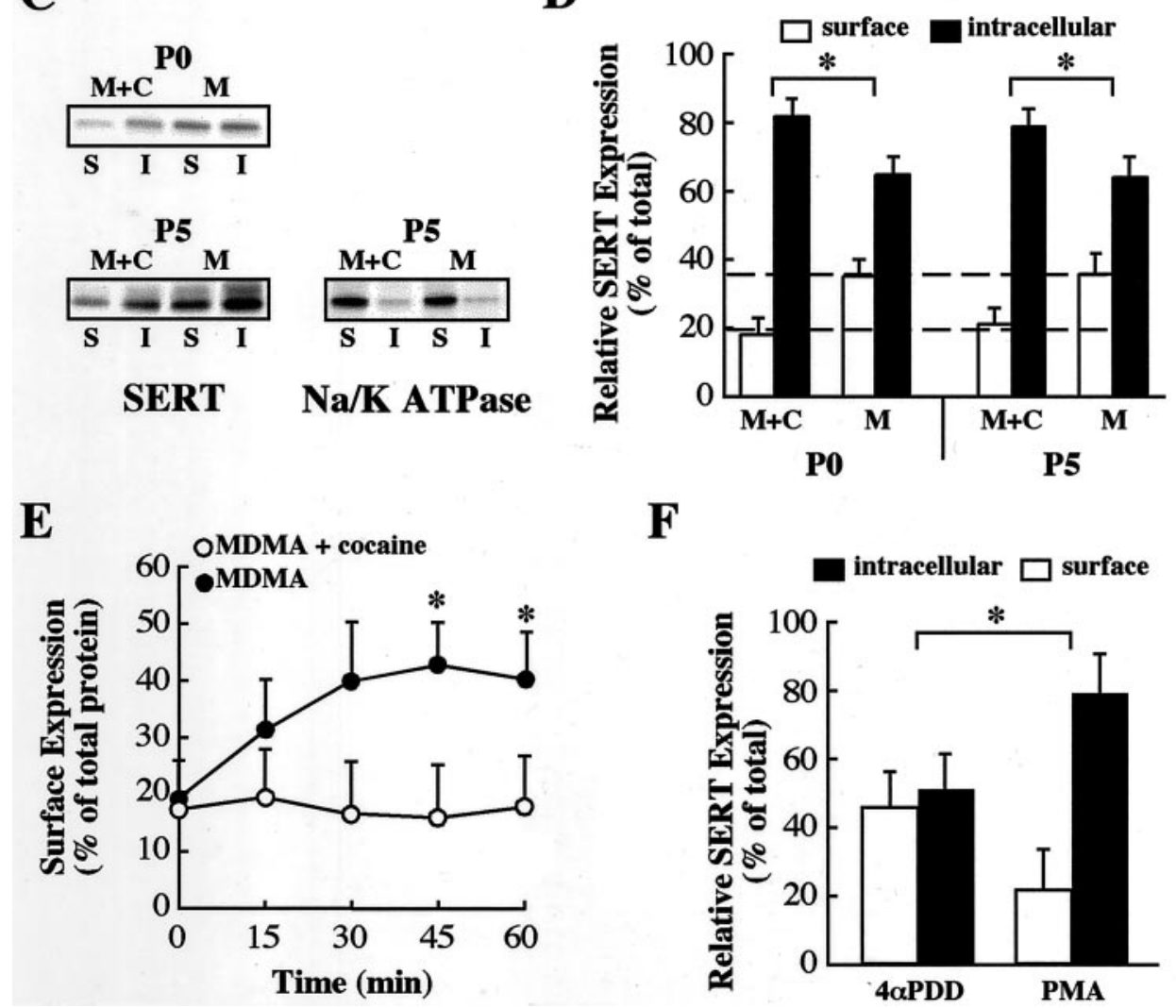

B

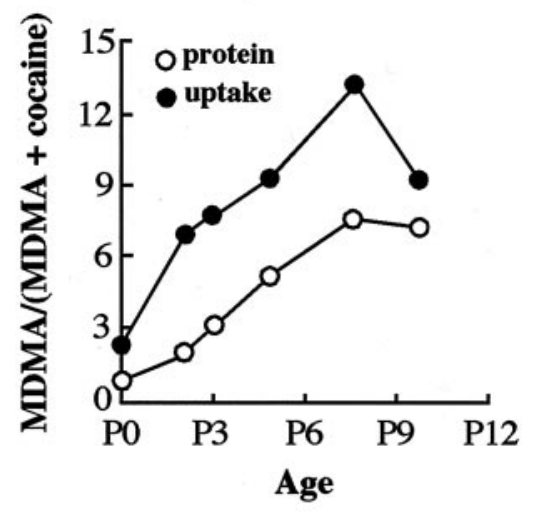

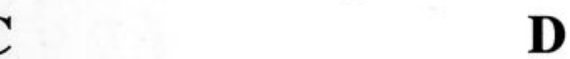

F

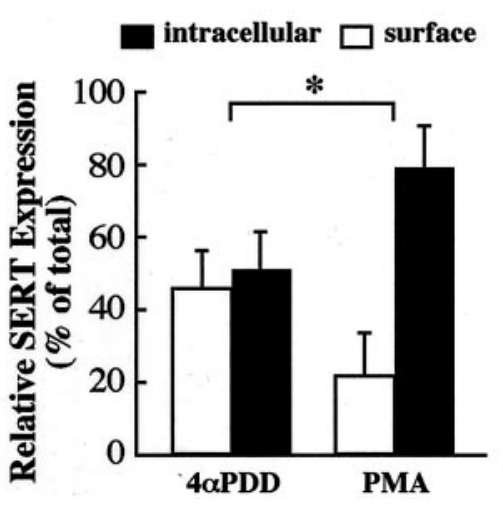

compared with cultures treated with MDMA and cocaine. However, although the time course of 5-HT uptake and SERT immunoreactivity were comparable, at all ages the magnitude of 5-HT uptake was greater than that predicted from the changes seen in protein levels (Fig. $3 B$ ). For example, at P3, the ratio of immunoreactivity in MDMA-treated cultures to that of MDMA plus cocaine-treated cultures was $\sim 3$. For 5 -HT uptake, this ratio was $>7$. These data suggested that MDMA was not only affecting total SERT protein levels but the fraction of SERT that was functional.

Neurotransmitter transporters can be regulated not only by altering rates of flux through the transporter (Deken et al., 2000), but also by altering the subcellular distribution of transporter protein between intracellular sites and the plasma membrane (for review, see Beckman and Quick, 1998). This is true for SERT (Qian et al., 1997; Ramamoorthy and Blakely, 1999). The relative increase in 5-HT uptake compared with SERT protein levels was consistent with the hypothesis that, in the presence of MDMA, a greater proportion of SERT is localized to the plasma membrane. To test this hypothesis, we performed surface biotinylation experiments and examined the subcellular distribution of SERT (Fig. 3C). Cultures were treated with MDMA alone or MDMA in the presence of cocaine, and examined at P0 or P5. At both ages, and in each treatment condition, the majority of SERT was found intracellularly. However, in cocaine-treated cultures, $\sim 20 \%$ of SERT was surface biotinylated; in MDMA-treated cultures, the amount of SERT found in the biotinylated fraction increased to $\sim 40 \%$ (Fig. $3 D$ ). These drugs had no effects on the subcellular distribution of the $\mathrm{Na} / \mathrm{K}$ ATPase (Fig. 3C). The approximate twofold increase in surface SERT expression would account for the increase in uptake compared with total protein levels (Fig. 3B). These data suggest that MDMA has two effects on regulating SERT: it increases total SERT protein levels and it increases the relative amount of SERT on the plasma membrane. 
The effect of SERT substrates on upregulation of total protein levels appeared to require prolonged drug treatment (Fig. 2D). To determine whether the substrate-mediated effects on SERT redistribution occurred on a similar time scale, we acutely applied MDMA alone or in combination with cocaine to previously untreated P3 cultures (Fig. 3E). The MDMA-induced subcellular redistribution of SERT occurred within minutes. This time course is similar to results seen with substrate-mediated redistribution of GABA transporters (Bernstein and Quick, 1999). Another feature of many neurotransmitter transporters is its internalization in the presence of activators of protein kinase $\mathrm{C}$ (PKC) (Beckman and Quick, 1998). In expression systems, SERT substrates prevent PKC phosphorylation of SERT and increase the relative amount of surface SERT expression (Ramamoorthy and Blakely, 1999). To determine whether a similar mechanism was occurring in neurons, MDMA-treated cultures were also treated with either the phorbol ester PMA or an inactive control (4 $\alpha$ PDD). We reasoned that the addition of PMA would increase the likelihood that SERT would be PKC phosphorylated. The addition of PMA prevented the MDMA-mediated redistribution of SERT to the plasma membrane, as assessed by surface biotinylation (Fig. $3 F$ ).

\section{DISCUSSION}

Neonatal serotonin (5-HT) depletion results in changes in neuronal morphology (Bennett-Clarke et al., 1994) and decreased synaptic density (Mazer et al., 1997). Conversely, 5-HT during early postnatal development modulates synaptic plasticity (Kojic et al., 1997; Edagawa et al., 2001), activity (Rhoades et al., 1994), and maturation (Borella et al., 1997). In rats, experimental alterations in 5-HT levels during the early postnatal period also results in characteristic behavioral syndromes (Breese et al., 1978; Tricklebank, 1985) and long-term cognitive impairments (Nyakas et al., 1994; Buwalda et al., 1995; Whitaker-Azmitia et al., 1995). In addition, disruption of normal serotonergic signaling via gene knock-out of monoamine oxidase, 5-HT1B receptors, or 5-HT transporters (SERTs) reveals that 5-HT systems play a crucial role in the formation and segregation of somatosensory (Blue et al., 1991; Cases et al., 1996) and visual system sensory maps (Upton et al., 1999; Salichon et al., 2001). A significant serotonergic input to cortex during development comes from thalamocortical neurons that borrow 5-HT (Lebrand et al., 1996; Bruning and Liangos, 1997). The borrowing of 5-HT during early postnatal development is attributable to the transient expression of SERT and the vesicular amine transporter (Lebrand et al., 1998). In the present experiments, we show that 5-HT and psychostimulants regulate the time course and peak expression of SERT in thalamocortical neurons. These data suggest that some of the actions of psychostimulants in neocortical development may be attributable to alterations in SERT expression and concomitant changes in 5-HT signaling.

The ability of endogenous SERT to be differentially regulated by psychostimulants that act as either antagonists or substrates of SERT is consistent with evidence from heterologous expression systems (Ramamoorthy and Blakely, 1999). This regulation occurs via three mechanisms: a change in the total amount of SERT protein, a change in the time course of SERT expression, and a redistribution of SERT between intracellular stores and the plasma membrane. Yet to be determined are the molecular processes that underlie these mechanisms. The substrate-induced upregulation of total protein expression levels would indicate regulation of protein synthesis. Substrates are clearly not the only signal for controlling SERT protein levels because SERT disappears from these neurons even in the presence of substrate. The regulation of SERT expression time course appears to require the sustained presence of substrate because removal of substrate results in a more rapid rate of disappearance of cellular SERT protein. The much more rapid substrate-induced increase of SERT to the plasma membrane suggests a mechanism by which rates of SERT internalization or externalization are altered.

Although regulation of transporter expression is a common feature of plasma membrane transporters, the details of how agonists and antagonists affect transporter function appear to be transporter-specific. Similarly to SERT, heterologously expressed norepinephrine transporters (NETs) are downregulated after chronic treatment with the NET antagonist desipramine, perhaps through changes in protein expression and/or transporter turnover (Zhu et al., 1998). In contrast to SERT, the NET agonists norepinephrine and amphetamine also cause decreases in expression (Zhu et al., 2000). In heterologously expressed dopamine transporters (DATs), DAT agonists dopamine and amphetamine cause an net accumulation of DAT intracellularly; this internalization is blocked by the DAT antagonist cocaine (Saunders et al., 2000). In endogenously expressed GABA transporters, agonists cause a net externalization of the transporter, and antagonists cause a net internalization; however, there is no effect on total transporter protein levels (Bernstein and Quick, 1999). The mechanisms and biological relevance underlying these different effects of transporter substrates and antagonists on transporter expression is not known. However, the ability of amine transporters to be differentially regulated by psychostimulants is important both in understanding the molecular basis of drug abuse and in the design of strategies to intervene in the abuse process.

\section{REFERENCES}

Beckman ML, Quick MW (1998) Neurotransmitter transporters: regulators of function and functional regulation. J Membr Biol 164:1-10.

Bennett-Clarke CA, Leslie MJ, Chiaia NL, Rhoades RW (1993) Serotonin $1 \mathrm{~B}$ receptors in the developing somatosensory and visual cortices are located on thalamocortical axons. Proc Natl Acad Sci USA 90:153-157.

Bennett-Clarke CA, Leslie MJ, Lane RD, Rhodes RW (1994) Effect of serotonin depletion on vibrissa-related patterns of thalamic afferents in the rat's somatosensory cortex. J Neurosci 14:7594-7607.

Bernstein EM, Quick MW (1999) Regulation of $\gamma$-aminobutyric acid (GABA) transporters by extracellular GABA. J Biol Chem 274:889-895.

Blakely RD, Berson HE, Fremeau RT, Caron MG, Peek MM, Prince HK, Bradley CC (1991) Cloning and expression of a functional serotonin transporter from rat brain. Nature 354:66-70.

Blue ME, Erzurumlu RS, Jhaveri S (1991) A comparison of pattern formation by thalamocortical and serotonergic afferents in the rat barrel field cortex. Cereb Cortex 1:380-389.

Borella A, Bindra M, Whitaker-Azmitia PM (1997) Role of the 5-HT1A receptor in development of the neonatal rat brain: preliminary behavioral studies. Neuropharmacology 36:445-450.

Breese GR, Vogel RA, Mueller RA (1978) Biochemical and behavioral alterations in developing rats treated with 5,7-dihydroxytryptamine. J Pharmacol Exp Ther 205:587-595.

Bruning G, Liangos O (1997) Transient expression of the serotonin transporter in the developing mouse thalamocortical system. Acta Histochem 99:117-121.

Buwalda B, Nyakas C, Vosselman HJ, Luitem PGM (1995) Effects of early postnatal anoxia on adult learning and emotion in rats. Behav Brain Res 67:85-90.

Buznikov GA, Shmukler YB (1981) Possible role of "prenervous" neurotransmitters in cellular interactions of early embryogenesis: a hypothesis. Neurochem Res 6:55-68.

Cases O, Vitalis T, Seif I, De Maeyer E, Sotelo C, Gaspar P (1996) Lack of barrels in the somatosensory cortex of monoamine oxidase A-deficient mice: role of serotonin excess during the critical period. Neuron 16:297-307.

Corey JL, Davidson N, Lester HA, Brecha N, Quick MW (1994) Protein kinase $\mathrm{C}$ modulates the activity of cloned $\gamma$-aminobutyric acid trans- 
porter expressed in Xenopus oocytes via regulated subcellular redistribution of the transporter. J Biol Chem 269:14759-14767.

D'Amato RJ, Blue ME, Largent BL, Lynch DR, Ledbetter DJ, Molliver ME, Snyder SH (1987) Ontogeny of the serotonergic projection to rat neocortex: transient expression of a dense innervation to primary sensory areas. Proc Natl Acad Sci USA 84:4322-4326.

Davis KE, Straff DJ, Weinstein EA, Bannerman PG, Correale DM, Rothstein JD, Robinson MB (1998) Multiple signaling pathways regulate cell surface expression and activity of the excitatory amino acid carrier 1 subtype of glu transporter in C6 glioma. J Neurosci 18:2475-2585.

Deken S, Beckman M, Boos L, Quick MW (2000) Permeation through GABA transporters is regulated by syntaxin 1A. Nat Neurosci 3:998-1003.

Dori I, Dinopoulos A, Blue ME, Parnavelas JG (1996) Regional differences in the ontogeny of the serotonergic projection to the cerebral cortex. Exp Neurol 138:1-15.

Edagawa Y, Saito H, Abe K (2001) Endogenous serotonin contributes to a developmental decrease in long-term potentiation in the rat visual cortex. J Neurosci 21:1532-1537.

Hansson SR, Mezey E, Hoffman BJ (1998) Serotonin transporter messenger RNA in the developing rat brain: early expression in serotonergic neurons and transient expression in non-serotonergic neurons. Neuroscience 83:1185-1201.

Hoffman BJ, Mezey E, Brownstein MJ (1991) Cloning of a serotonin transporter affected by antidepressants. Science 254:579-580.

Jayanthi LD, Ramamoorthy S, Mahesh VB, Leibach FH, Ganapathy V (1994) Calmodulin-dependent regulation of the catalytic function of the human serotonin transporter in placental choriocarcinoma cells. J Biol Chem 269:14424-14429.

Kojic L, Gu Q, Douglas RM, Cynader MS (1997) Serotonin facilitates synaptic plasticity in kitten visual cortex: an in vitro study. Brain Res Dev Brain Res 101:299-304.

Lauder JM (1993) Neurotransmitters as growth regulatory signals: role of receptors and second messengers. Trends Neurosci 16:233-239.

Launay JM, Bondoux D, Oset-Gasque MJ, Emami S, Mutel V, Haimart M, Gespach C (1994) Increase of human platelet serotonin uptake by atypical histamine receptors. Am J Physiol 266:R526-R536.

Lebrand C, Cases O, Aldebrecht C, Doye A, Alvarez C, El-Mestikawy S, Seif I, Gaspar P (1996) Transient uptake and storage of serotonin in developing thalamic neurons. Neuron 17:823-835.

Lebrand C, Cases O, Wehrle R, Blakely RD, Edwards RH, Gaspar P (1998) Transient developmental expression of monoamine transporters in the rodent forebrain. J Comp Neurol 401:506-524.

Mazer C, Muneyyirci J, Taheny K, Raio N, Borella A, Whitaker-Azmitia P (1997) Serotonin depletion during synaptogenesis leads to decreased synaptic density and learning deficits in the adult rat: a possible model of neurodevelopmental disorders with cognitive deficits. Brain Res 760:68-73.

Miller KJ, Hoffman BJ (1994) Adenosine $\mathrm{A}_{3}$ receptors regulate serotonin transport via nitric oxide and cGMP. J Biol Chem 269:27351-27356.

Nyakas C, Nuwalda B, Kramers RKJ, Traber J, Luiten PGM (1994)
Postnatal development of hippocampal and neocortical cholinergic and serotonergic innervation in rat: effects of nitrite-induced prenatal hypoxia and nimodipine treatment. Neuroscience 39:541-559.

Qian Y, Galli A, Ramamoorthy S, Risso S, DeFelice LJ, Blakely RD (1997) Protein kinase $\mathrm{C}$ activation regulates human serotonin transporters in HEK-293 cells via altered cell surface expression. J Neurosci 17:45-57.

Ramamoorthy S, Blakely RD (1999) Phosphorylation and sequestration of serotonin transporters differentially modulated by psychostimulants. Science 285:763-766.

Ramamoorthy S, Giovanetti E, Qian Y, Blakely RD (1998) Phosphorylation and regulation of antidepressant-sensitive serotonin transporters. J Biol Chem 273:2458-2466.

Rhoades RW, Bennett-Clarke CA, Shi M-Y, Mooney RD (1994) Effects of 5-HT on thalamocortical synaptic transmission in the developing rat. J Neurophysiol 72:2438-2450.

Rudnick G, Wall SC (1992) The molecular mechanism of "ecstasy" [3,4methylenedioxy-methamphetamine (MDMA)]: serotonin transporters are targets for MDMA-induced serotonin release. Proc Natl Acad Sci USA 89:1817-1821.

Salichon N, Gaspar P, Upton AL, Picaud S, Hanoun N, Hamon M, De Maeyer E, Murphy DL, Mossner R, Lesch KP, Hen R, Seif I (2001) Excessive activation of serotonin (5-HT) 1B receptors disrupts the formation of sensory maps in monoamine oxidase A and 5-HT transporter knock-out mice. J Neurosci 21:884-896.

Saunders C, Ferrer JV, Shi L, Chen J, Merrill G, Lamb ME, LeebLundberg LM, Carvelli L, Javitch JA, Galli A (2000) Amphetamineinduced loss of human dopamine transporter activity: an internalization-dependent and cocaine-sensitive mechanism. Proc Natl Acad Sci USA 97:6850-6855.

Tricklebank MD (1985) The behavioral response to 5-HT receptor agonists and subtypes of the central 5-HT receptor. Trends Pharmacol Sci 3:403-406.

Upton AL, Salichon N, Lebrand C, Ravary A, Blakely R, Seif I, Gaspar $P$ (1999) Excess of serotonin (5-HT) alters the segregation of ipsilateral and contralateral retinal projections in monoamine oxidase A knock-out mice: possible role of 5-HT uptake in retinal ganglion cells during development. J Neurosci 19:7007-7024.

Whitaker-Azmitia PM, Borella A, Raio N (1995) Serotonin depletion in the adult rat causes loss of dendritic marker MAP-2. A new animal model of schizophrenia? Neuropharmacology 12:269-272.

Yura A, Kiuchi Y, Uchikawa T, Uchida J, Yamazaki K, Oguchi K (1996) Possible involvement of calmodulin-dependent kinases in $\mathrm{Ca}^{2+}$. dependent enhancement of $\left[{ }^{3} \mathrm{H}\right] 5$-hydroxytryptamine uptake in rat cortex. Brain Res 738:96-102.

Zhu MY, Blakely RD, Apparsundaram S, Ordway GA (1998) Downregulation of the human norepinephrine transporter in intact 293hNET cells exposed to desipramine. J Neurochem 70:1547-1555.

Zhu MY, Shamburger S, Li J, Ordway GA (2000) Regulation of the human norepinephrine transporter by cocaine and amphetamine. J Pharmacol Exp Ther 295:951-959. 